\title{
IMPLICACIONES DE LA INTELIGENCIA EMOCIONAL EN LOS ESTILOS DE LIDERAZGO
}

\section{IMPLICATIONS OF EMOTIONAL INTELLIGENCE IN LEADERSHIP STYLES}

\author{
MARÍA AUXILIADORA VARGAS VALDIVIEZO
}

Universidad Católica de Santiago de Guayaquil.maria.vargas06@cu.ucsg.edu.ec

RESUMEN

Este trabajo parte de una revisión de la literatura acerca de los conceptos, relaciones e implicaciones de la inteligencia emocional y los estilos de liderazgo describe algunas de las contradicciones y alcances de estos dos conceptos. En conocimiento de que las emociones son uno de los aspectos más complejos de los seres humanos y que éstos pueden influir en las relaciones interpersonales, debido a que guían nuestros pensamientos y comportamientos. Estos pensamientos y comportamientos bien manejados determinan las formas de reaccionar de las personas, lo que evidencia habilidad para afrontar situaciones críticas o de presión en la vida cotidiana, es una muestra de inteligencia emocional. De la literatura se deduce que las personas con mayor inteligencia emocional poseen una ventaja natural para manejar situaciones y que perciben de forma más ágil las manifestaciones emocionales de su entorno lo que les facilita las interacciones, obteniendo con la aceptación de las personas lo que hace posible que el liderazgo que ejercen sea admitido casi sin resistencia.

PALABRAS CLAVE: emociones, inteligencia emocional, liderazgo, estilo de liderazgo. This work starts from a literature review about concepts, relations and emotional intelligence implications, describe some contradictions and those two concepts scope. In knowledge that emotions are one of the most complex aspects from human being and that they can influence on interpersonal relationships as they guide our thoughts and behaviors. These thoughts and behaviors well managed determine the different ways of people reaction, which shows the ability to face critical or pressure situations in everyday life, and also shows emotional intelligence. Of the literature is deduced that people with greater emotional intelligence have a natural advantage to handle situations and perceive, in a more agile way, emotional manifestations from their environment what makes easier the interactions development, getting people's acceptance what allows leadership to be admitted almost without opposition.

KEYWORDS: emotions, emotional intelligence, leadership, leadership style. 
INTRODUCCIÓN

En un mundo globalizado en el que el cambio y la transformación son las mayores constantes, la organización para coexistir entre los demandantes requerimientos de la sociedad requiere de gente con capacidades y competencias que hagan posible su sostenibilidad en el tiempo a través de la toma de decisiones (Coldman, Scott, \& Follman, 2015; Heracleous, 1998). "La producción de bienes y servicios ya no se realiza por personas que trabajan de manera aislada e individual. Cuanto más industrializada es una sociedad, tanto más depende de las organizaciones para satisfacer sus necesidades y aspiraciones (Chiavenato et al., 2011, p. 2). Consecuentemente, las organizaciones deben ser guiadas por líderes que ejecuten su tarea de forma estratégica y que posean las habilidades cognitivas del pensamiento crítico (Lovelace, Eggers, \& Dyck, 2016). De la misma forma el gerente debe poseer liderazgo y según D’Alessio (2010) señaló el liderazgo es un proceso que involucra una visión y pensamiento de largo plazo donde es innato la mejora continua.

Anteriormente se tenía una perspectiva y se pensaba que ejercer liderazgo era sinónimo de autoridad y dominio a los subordinados, pero se ha ido evolucionando en referencia a esta temática debido a que se la ha abordado de forma integral permitiendo comprender de forma más pragmática los comportamientos del liderazgo; se ha revisado el concepto desde la perspectiva de que al estar relacionada con el ser humano, debe entenderse como una realidad compleja. En las dos últimas décadas, las organizaciones han visto un cambio en lo referente a los estilos de gestión y de liderazgo, se han enfocado más a lograr la satisfacción del cliente y a la necesidad de trabajar en equipo. Esto ha hecho imprescindible que los líderes, ejecutivos y gerentes desarrollen habilidades para entender y trabajar con otros. Lo que ha evidenciado que las competencias de la inteligencia emocional sean parte de las características de los líderes sobresalientes.

La línea de tiempo histórica de las inteligencias muestra que este no es un concepto nuevo, pero que con el tiempo ha obtenido un acuerdo general como elemento clave del éxito en los lugares de trabajo.

DESARROLLO

A partir de una revisión de la literatura acerca de los conceptos de inteligencia emocional y los estilos de liderazgo, se intenta describir algunas de las relaciones e implicaciones de estos dos conceptos; para ello se hace preciso definir la inteligencia emocional; una de las primeras conceptualizaciones en el mundo científico la realiza Thorndike (1920) quien identificó a la inteligencia social como una dimensión de la inteligencia; su definición la describe como la capacidad de comprender y gestionar los hombres y las mujeres, niños y niñas, para actuar con sabiduría en las relaciones humanas. Esta definición hace referencia a la capacidad del individuo para entender y manejar sus habilidades en las relaciones interpersonales, que es en esencia como se percibe a la inteligencia emocional en la actualidad (Dabke, 2016).

Mayer y Salovey (1997) afirmaron que la inteligencia emocional es la capacidad de apreciar, utilizar, entender y manejar las emociones, por tanto se puede decir que es una habilidad a través de la cual se puede distinguir sentimientos y emociones que permiten comprender y guiar el pensamiento y la actuación de los individuos; también se dice que la inteligencia emocional es la forma de expresión que tienen las personas para exteriorizar sus agrados y desagrados; a la vez que se podría definir también como la forma en que se afronta los retos que nos impone la vida a diario (Van Rooy \& Viswesvaran, 2004). Otros autores también han definido la inteligencia emocional, como Mayer, Salovey y Caruso (2004): «la capacidad de razonar acerca de las emociones y las emociones para mejorar el pensamiento. Incluye las habilidades para percibir con precisión las emociones... con el fin de promover el crecimiento emocional e intelectual». De la misma forma Mayer y Salovey, 1993 "la inteligencia emocional es un tipo de inteligencia social» (citado por D’Alessio, 2010), según Trujillo y Rivas (2005) el concepto propuesto por Mayer y Salovey, tuvo su fundamento en lo expuesto por Gardner en su teoría de inteligencias múltiples que a la vez tiene origen en la ley del efecto de Thorndike expuesta en su tesis doctoral.

Según Joseph, Jin, Newman y O’Boyle (2015) indicaron que la inteligencia emocional "está considerada en la actualidad por la comunidad empresarial como una herramienta profesional ampliamente aceptada para la contratación, capacitación, desarrollo de liderazgo y formación de equipos”. Según los creadores del concepto de inteligencia emocional "está formada por meta-habilidades que pueden ser categorizadas en cinco competencias: (a) conocimiento de las propias emociones, (b) capacidad para controlar emociones, (c) capacidad de motivarse a sí mis- 
mo, (d) reconocimiento de emociones ajenas y (e) control de las relaciones" (citado por Trujillo \& Rivas, 2005).

Se hace preciso señalar que este tema ha sido tratado por muchos años y desde la perspectiva de muchas disciplinas entre las que podemos citar a la psicología, en las ciencias médicas de forma específica en la neurología, en la educación, en la comunicación y desde luego en el campo de la administración, desde la relación con otros conceptos como el liderazgo.

De acuerdo a Goleman (2006) mencionó en su texto las personas que tienen un elevado coeficiente intelectual no tienen garantizado el éxito en su vida personal cotidiana y profesional; el autor dice que el éxito o fracaso dependen de la inteligencia emocional que como ya hemos visto está ligada a la forma en que manejemos nuestras emociones, puede conducirnos a mejorar nuestro desempeño profesional y que la incorrecta administración de las emociones puede tener efectos negativos, pero porque nos beneficia un buen manejo de las emociones, nos ayuda a tener control para afrontar las presiones y evita que el individuo se sienta frustrado, además trae consigo una mejor relación con las personas que comparten el ambiente; se podría decir que nos ayuda a mantener un sentido de equilibrio.

Por ello se hace preciso entender el liderazgo, de acuerdo con Burns "el liderazgo es el proceso recíproco de movilizar, por personas con ciertos motivos y valores, varios recursos económicos, políticos, y otros, en un contexto de competencia y conflicto, para lograr las metas independiente o mutuamente abrazadas por líderes y seguidores" (citado por D’Alessio, 2010, p. 7); de la misma forma se debe revisar la literatura acerca del liderazgo y los estilos de liderazgo, según Burns (1978) estableció la diferencia entre el liderazgo transaccional y transformacional, lo que según Ashkanasy y Tse, y George, 2000 "es uno de los rasgos más distintivos del liderazgo y específicamente del liderazgo transformacional" (citado por Føllesdal \& Hagtvet, 2013, p. 748).

Ahora bien después de haber analizado los conceptos de inteligencia emocional y el liderazgo y sus estilos transaccional y transformacional, según Ashkanasy y Tse , 2000 «el liderazgo transformacional es intrínsecamente relacionado con la inteligencia emocional» (citado por Føllesdal \& Hagtvet, 2013), según otro estudio realizado por Ashkanasy, Hartel, y Daus, 2002 "las características del liderazgo transformacional se asemejan a los componentes de la inteligencia emocional» (citado por Føllesdal \& Hagtvet, 2013, p. 749). De acuerdo a lo antes expuesto se puede decir que contrariamente a lo que se piense acerca de la inteligencia emocional y su relación con el liderazgo, cuando se agregan los factores de la personalidad como parte de los constructos para evidenciar la relación, esta se bloquea y se evidencia discrepancias con lo expuesto, lo que permite pensar que la personalidad del líder no tiene relación con la inteligencia emocional que posea para el manejo de sus emociones y de su entorno.

A partir de las cualidades que Goleman (2006) mencionó cinco características de la inteligencia emocional que a su vez las agrupó en las competencias personales las cuales engloban a las siguientes : (a) autoconciencia, se refiere a la capacidad de reconocer las fortalezas y las debilidades que posee; (b) autorregulación, hace referencia a la capacidad de control, flexibilidad y compromiso; (c) motivación, mantener constancia en un elevado desempeño para conseguir transmitirlo a los subordinados y las competencias sociales en las que agrupó a: (1) empatía, se dice de la capacidad de ponerse en los zapatos de los demás y (2) habilidades sociales, esto se refiere a la capacidad de relacionarse y comunicarse; en estas breves descripciones el autor ha visto como las cualidades mencionadas se han relacionado con las cualidades del liderazgo.

El liderazgo de acuerdo a la definiciones antes descritas se lo concibe como un proceso emocional a través del cual el líder busca motivar actitudes en los empleados a su cargo para lograr los resultados más satisfactorios para la organización; además este proceso conlleva que evocar sentimientos en los empleados que fortalezcan el clima organizacional. Según Sadri (2012) indicó que los estudios han provisto importantes argumentos a favor y en contra acerca de que los componentes de la inteligencia emocional se pueden integrar a las prácticas de liderazgo debido a los enlaces entre el liderazgo y cuatro de los cinco componentes del modelo de inteligencia emocional de Coleman.

Existe otro estudio que ha analizado a la inteligencia emocional desde otra perspectiva la relación entre la inteligencia emocional y el estilo de liderazgo transformacional, según Lindebaum y Cartwright (2010) indicaron en su estudio que la relación entre la inteligencia emocional y liderazgo transformacional se 
vuelva significativa y es fuertemente dependiente cuando se desarrolla en un contexto favorable. En este sentido se puede indicar que esta relación en un contexto favorable va a significar que el ambiente laboral sea más agradable lo que tiene efecto en la productividad debido a que se evidencia un buen manejo del estrés, logrando un clima laboral adecuado generando un impacto positivo en el desempeño. Según Calcina (2014), la influencia del clima sobre el desempeño, implica considerar tanto las repercusiones del clima sobre las actitudes y comportamientos (p.23).

Por otra parte, de acuerdo a Chatterjee y Kulakli, (2015) indicaron que después de haber analizado numerosos estudios y como resultado de un meta-análisis se puede inferir que los comportamientos de liderazgo transformacional están correlacionados con más fuerza con la inteligencia emocional que los comportamientos de liderazgo transaccional. Para llegar a esta deducción se revisó la conexión del liderazgo con la emoción, Soskin y Megerian (1999) estudiaron la relación del comportamiento de liderazgo transformacional, la inteligencia emocional y la efectividad del líder (citado por Chatterjee \& Kulakli, 2015). También George y Bettenhausen (1990 ) y George (1995) investigaron algunas de las posibles ventajas del estado de ánimo y los sentimientos del líder y cómo influyen en la eficacia del liderazgo en situaciones complejas (citado por George, 2000).

CONCLUSIONES

Después de haber realizado esta breve revisión de literatura sobre la temática, se pueda inferir que las emociones tienen un importante rol en el escenario de nuestra vida cotidiana; de la misma forma, el tener un elevado coeficiente intelectual no es señal de inteligencia emocional; asimismo desde los aportes de los autores que introdujeron los términos de inteligencias múltiples, inteligencia emocional; como esta característica puede determinar las metas que una persona alcance, ya que al estar preparado para afrontar situaciones que nos retan, nos volvemos más competitivos y se desarrollan características deseables en un empleado de una organización.

Las compañías no deben perder de vista a este tipo de personas, porque seguramente en ellas se puede manifestar un líder que haga posible que las metas organizacionales e individuales de los empleados, se alineen generando condiciones favorables para todos; para los empleados, el reconocimiento moral y econó- mico por sus resultados para la organización, posicionamiento y competitividad debido al desempeño de su gente.

En la actualidad las empresas han adoptado mecanismos de recursos humanos en lo referente a la selección, capacitación y al desarrollo del personal, para lo cual han establecido pruebas para medir el coeficiente intelectual;otras para medir la inteligencia emocional para de esa forma asegurarse de enganchar a las personas más idóneas, sin perder de vista que la inteligencia "persé" no es garantía de que se logrará el éxito. Este desafío también ha sido asumido por las instituciones educativas que están incluyendo en sus flujos curriculares, asignaturas que potencien el desarrollo de inteligencia emocional como parte de la formación, debido a que las organizaciones demandan profesionales con habilidades en el manejo del personal de forma que se reduzcan al mínimo deseable las tensiones en el trabajo y se eleve el grado de desempeño.

\section{REFERENCIAS BIBLIOGRÁFICAS}

Burns, J. M. (1978). Leadership. New York: Harper \& Row.

Calcina, Y. C. (2014). El clima institucional y su incidencia en el desempeño laboral de los docentes de la Facultad de Ciencias Sociales de la Universidad Nacional del Altiplano y Facultad de Ciencias de la Educación Universidad Andina Néstor Cáceres Velásquez-Perú 2012. Comunic@ ción, 5(1), 22-29.

Chatterjee, A., \& Kulakli, A. (2015). An Empirical Investigation of the Relationship Between Emotional Intelligence, Transactional and Transformational Leadership Styles in Banking Sector. Procedia - Social and Behavioral Sciences, 210, 291-300. https://doi.org/10.1016/j. sbspro.2015.11.369

Chiavenato, I., Bárcenas Salas, M., Saleme Aguilar, M. M., Toledo Castellanos, M. Á., Mascaró Sacristán, P., Hano Roa, M. del C. E., \& Obón León, M. del P. (2011). Administración de recursos humanos: el capital humano de las organizaciones (9na Ed.). México D.F.: McGrawHill.

Dabke, D. (2016). Impact of Leaders Emotional Intelligence and Transformational Behavior on Perceived Leadership Effectiveness: A Multiple Source View. Business Perspectives and Research, 4(1), 27-40. https://doi. org $/ 10.1177 / 2278533715605433$ 
D’Alessio, F. (2010). Liderazgo y atributos Lovelace, K. J., Eggers, F., \& Dyck, L. R. (2016). I gerenciales: una visión global y estratégica. Do and I Understand: Assessing the Utility Lima; Naucalpan de Juárez: CENTRUM ; Pearson Educación.

Føllesdal, H., \& Hagtvet, K. (2013). Does emotional intelligence as ability predict transformational leadership? A multilevel approach. The Leadership Quarterly, 24(5), 747-762. https://doi. org/10.1016/j.leaqua.2013.07.004

George, J. M. (2000). Emotions and leadership: The role of emotional intelligence. Human Relations, 53(8), 1027-1055. https://doi. org/10.1177/0018726700538001

Goldman, E. F., Scott, A. R., \& Follman, J. M. (2015). Organizational practices to develop strategic thinking. Journal of Strategy and Management, 8(2), 155-175. https://doi.org/10.1108/JSMA-012015-0003

Goleman, D. (2006). Emotional intelligence (Bantam 10th anniversary hardcover ed). New York: Bantam Books.

Heracleous, L. (1998). Strategic thinking or strategic planning? Long Range Planning, 31(3), 481-487. https://doi.org/10.1016/S0024-6301(98)80015-0

Joseph, D. L., Jin, J., Newman, D. A., \& O’Boyle, E. H. (2015). Why does self-reported emotional intelligence predict job performance? A metaanalytic investigation of mixed EI. Journal of Applied Psychology, 100(2), 298-342. https://doi. org/10.1037/a0037681

Lindebaum, D., \& Cartwright, S. (2010). A Critical Examination of the Relationship between Emotional Intelligence and Transformational Leadership. Journal of Management Studies, 47(7), 1317-1342. https://doi.org/10.1111/j.14676486.2010.00933.x of Web-Based Management Simulations to Develop Critical Thinking Skills. Academy of Management Learning \& Education, 15(1), 100121. https://doi.org/10.5465/amle.2013.0203

Mayer, J. D., \& Salovey, P. (1997). What is Emotional Intelligence? In Emotional development and emotional intelligence: educational implications (1st ed). New York: Basic Books.

Mayer, J. D., Salovey, P., \& Caruso, D. R. (2004). TARGET ARTICLES: "Emotional Intelligence: Theory, Findings, and Implications." Psychological Inquiry, 15(3), 197-215. https:// doi.org/10.1207/s15327965pli1503_02

Sadri, G. (2012). Emotional Intelligence and Leadership Development. Public Personnel Management, 41(3), 535-548.

Thorndike, E. (1920). Intelligence and its uses. Harper's Magazine, 140, 227-235.

Trujillo Flores, M. M., \& Rivas Tovar, L. A. (2005). The origins, evolution and construction of models of emotional intelligence. Innovar, 15(25), 9-24.

Van Rooy, D. L., \& Viswesvaran, C. (2004). Emotional intelligence: A meta-analytic investigation of predictive validity and nomological net. Journal of Vocational Behavior, 65(1), 71-95. https://doi. org/10.1016/S0001-8791(03)00076-9 